\title{
DIAGNÓSTICO DO USO DE AGROTÓXICOS POR TOMATICULTORES DO MUNICÍPIO DE SÃO JOSÉ DE UBÁ, RJ
}

\author{
Jaomara Nascimento da Silva ${ }^{1}$, Tainá Costa Araújo² $^{2}$, Niraldo José Ponciano ${ }^{3}$, Claudio Luiz Melo de Souza ${ }^{3}$
}

\begin{abstract}
RESUMO - O município de São José de Ubá apresenta o maior volume de produção de tomate do Estado do Rio de Janeiro. A horticultura, no geral, é responsável por grande parte da utilização de agrotóxico, devido a grande susceptibilidade à pragas e doenças. $\mathrm{O}$ objetivo desse trabalho foi avaliar a utilização de agrotóxicos realizado pelos produtores de São José de Ubá. Foram entrevistados 55 tomaticultores, utilizando-se um questionário para identificar o perfil do produtor e da produção, assim como aspectos relacionados ao manejo de pragas e doenças e as formas de aplicações agrotóxicos. A tomaticultura é a principal atividade de São José de Ubá, e se característica como uma pequena atividade rural familiar, com atividades realizadas por familiares e vizinhos. Cerca de 24 produtos (75\% inseticidas e $25 \%$ fungicidas) são usados de forma indiscriminada: sem necessidade, sem respeitar período de carência, sem assistência técnica durante todo o ciclo da cultura e sem o uso do EPI de forma completa e correta. Esses relatos refletem a necessidade de difusão de conhecimentos desses produtos que além de diminuir os custos de produção trará menos impacto aos agricultores, consumidores e ao meio ambiente.
\end{abstract}

Palavras chave: equipamento de proteção individual, manejo, Solanum lycopersicum.

\section{THE DIAGNOSES FOR PESTICES USES BY THE TOMATO FARMERS IN THE COUNTY OF SÃO JOSÉ DE UBÁ, RJ}

\begin{abstract}
The county of São José de Ubá is the largest tomato producer in the state of Rio de Janeiro. In general, the horticulture is responsible for most part of the pesticide utilization, due to the high susceptibility to pests and diseases. The objective of this project was to analyse the use of pests and diseases realized by the producers from São José de Ubá. It was interviewed 55 tomato farmers, using a questionnaire to identify its profiles and production, as well as their aspects associated with the handling of these pests and diseases and the way than they are applying the pesticides. Despite the city being responsible for half of the all-state production, the tomato production in São José de Ubá is characterized as a small family activity, with activities made by families and neighborhood. Around 24 different products (75\% insecticides and 25\% fungicides) are indiscriminately used: without requirement, not respecting grace period, lacking technical assistance during all cultivation cycle and without using the PPE (Personal protective equipment) in a complete and correct way. The pesticides utilization represents the greatest part of the costs, and most of the interviewed farmers believe that a production without those pesticides are a production without protection. This narrative emphasizes the need for diffusion of knowledge about those products beyond reduce the production costs bringing less impact for the farmers, consumers and the environment.
\end{abstract}

Keywords: management, personal protective equipment, Solanum lycopersicum.

\footnotetext{
1 Doutora em Produção Vegetal, UENF, RJ; e-mail: jaomarasilva@gmail.com

2 Estudante de Agronomia, UENF, RJ; e-mail: araujo.tainac@gmail.com

3 Engenheiro Agrônomo, Professor Doutor, UENF, RJ; e-mails: njponciano@gmail.com, claudiomelo.edu@gmail.com
} 


\section{INTRODUÇÃO}

A horticultura é responsável por grande parte da utilização de agrotóxicos no Brasil, através de pulverizações semanais para o controle de pragas e doenças em diversas culturas são realizadas (Almeida et al., 2009). Dentro deste ramo da agricultura, o tomate é a segunda hortaliça mais importante do Brasil, conforme Lima \& Campos (2014). E nesse contexto, a tomaticultura, um ramo de alto risco, principalmente pela grande susceptibilidade ao ataque de pragas e doenças, mas também pelas oscilações nos preços de mercado e grande exigências de insumos e serviços (Fernandes et al., 2007).

Para Reis Filho et al. (2009), tanto o cultivo de tomate em plantio "estaqueado" quanto no "rasteiro", exige grandes investimentos fitossanitários, sendo valido ressaltar que os danos causados pelo ataque dos patógenos podem reduzir o desenvolvimento, destruir tecidos, ou até mesmo matar a planta, o que reflete diretamente na baixa produtividade da cultura, ou danificar diretamente o produto final a ser colhido, reduzindo sua qualidade ou prejudicando sua aparência (Pereira, 2013).

O Brasil ocupa o nono lugar na produção mundial de tomate e o primeiro lugar com relação aos países da América do Sul (FAO, 2013), sendo a Região Sudeste, a maior produtora de tomate de mesa do país (Camargo e Filho, 2008). Nessa região observa-se que o Estado do Rio de Janeiro, nos últimos anos, tem tido uma ascensão nesse mercado, credenciando-o como o sexto maior produtor do país, com o município de São José de Ubá sendo responsável por cerca de 50\% da produção do Estado (IBGE, 2018).

O município de São José de Ubá apresenta o cultivo de tomate principalmente em sistemas convencionais, onde comumente o uso de agrotóxicos é um dos recursos mais utilizados pelos produtores rurais para tentar compensar a perda de produtividade provocada por pragas e doenças. Entretanto, é interessante apontar que muitas vezes, essa utilização de agrotóxicos é realizada sem atender o nível de dano econômico. E embora existam regulamentação quanto a comercialização e manejo dos agrotóxicos, é comum muitos produtores não respeitarem as regras impostas pela legislação, conforme relata Fernandes et al. (2012).

A agricultura convencional é comumente caracterizada por sua agressão ao meio ambiente ser cada vez maior, sendo este o grande desafio da humanidade (Duriguetto et al. 2011). Diante desse cenário, a utilização inadequada de agrotóxicos no sistema convencional de cultivo de tomate tem sido ponto fundamental na relação do produtor/consumidor, uma vez que representa a relação da qualidade dos frutos comercializados em relação à presença de possíveis resíduos de agrotóxicos. Assim, o trabalho teve por objetivo realizar um diagnóstico da utilização de agrotóxicos no cultivo de tomate em São José de Ubá, RJ.

\section{MATERIAL E MÉTODOS}

A área de estudo foi o município de São José de Ubá, localizado na Região Noroeste Fluminense. O municipio possui uma área de $251,36 \mathrm{~km}^{2}$, população de 7175 habitantes, com uma densidade demográfica de 28,63 hab. $/ \mathrm{km}^{2}$, sendo $56 \%$ distribuídos na zona rural (IBGE, 2018). São José de Ubá é dividido em cinco distritos: Barro Branco, Colosso, Brejo, Cambiocó e Santa Maria. Possui como municípios limítrofes: Santo Antônio de Pádua, Miracema, Itaperuna e Cambuci.

Os dados para realização deste trabalho foram obtidos por meio da aplicação de questionários semiestruturados a cinquenta e cinco tomaticultores, correspondendo a $30 \%$ do total de produtores de tomate neste ano. Para tal dimensionamento da amostra considerou-se o número produtores de tomate em sistemas convencionais atuando no município e assumiu-se $\mathrm{n}=$ N.(Z.e) ${ }^{2}$ (Stevenson, 2001). Para tanto, considerou-se a distribuição normal gaussiana com $95 \%$ de confiabilidade $(\mathrm{z}=1,96)$ e uma razão de $25 \%$ entre o erro padrão da população e o desvio padrão de sua estimativa $(\mathrm{e}=0,25)$, para o registro de 180 tomaticultores $(\mathrm{N})$ cadastrados no Centro de Abastecimento Regional (Ceasa) de São José de Ubá/RJ.

O processo metodológico inicial foi realizado através de entrevista com os tomaticultores dentro das propriedades. O questionário aplicado foi dividido em duas categorias de perguntas. A primeira categoria relacionada às características do produtor: idade, nível de escolaridade, tempo de atividade, mão de obra; e a segunda, relacionada ao uso de agrotóxicos: número de agrotóxicos, frequência de aplicação, classificação toxicológica, uso do Equipamento de Proteção Individual e conhecimento de produção de tomate sem agrotóxicos.

A pesquisa foi realizada entre os meses de maio a novembro de 2015 nos cinco distritos: Barro Branco (14), Santa Maria (10), Cambiocó (6), Colosso (10) e Brejo (15).

Os dados obtidos foram plotados no Microsoft Excel 2007, para escolha da forma gráfica de melhor compreensão das informações. Os resultados da presente pesquisa foram divulgados para os tomaticultores do município por intermédio de palestra realizada com a Secretaria de Agricultura e Emater Local de São José de Ubá. 


\section{RESULTADOS E DISCUSSÃO}

Em relação ao perfil do produtor e ao tipo de produção, a faixa etária dos entrevistados variou entre 19 a 60 anos, com a maioria (40\%) entre 39 a 48 anos. Em geral, 55\% dos agricultores são produtores de tomate há mais de 20 anos, o que caracteriza a produção de tomate como atividade tradicional do municipio. A maior parte dos agricultores $(62 \%)$ possuíam áreas de produção de tomate abaixo de 1 hectare, caracterizadas principalmente pelo pequeno agricultor familiar. Cerca de $80 \%$ dos tomaticultores contam com o número de 4 pessoas para a realização das tarefas agrícolas, sendo a mão de obra oriunda da família e vizinhos.

Sobre o uso dos agrotóxicos, cerca de 24 produtos diferentes são utilizados durante o cultivo do tomate no municipio de São José de Ubá, sendo $75 \%$ inseticidas e $25 \%$ fungicidas. Embora seja uma grande quantidade de produtos, ainda é um número inferior às 53 produtos de agrotóxicos encontrados no municipio vizinho (Cambuci) como descrito por Carvalho et al. (2016).

Observou-se que ao longo de todo ciclo da cultura há o uso de agrotóxicos na lavoura, no qual 75\% dos produtores fazem aplicação dos agrotóxicos entre duas a três vezes por semana. Os produtores relataram que as pulverizações já começam desde a semeadura, e ao transplantar, imediatamente já se começa a pulverizar a lavoura com agrotóxicos, semelhante ao encontrado por Reis Filho et al. (2009) em Goianopólis, onde o controle de pragas e doenças, é quase, exclusivamente, preventivo.

Do total dos produtores entrevistados, $80 \%$ fazem uso do Equipamento de Proteção Individual (EPI), entretanto, todos utilizam de maneira incompleta (Tabela 1). Os itens mais utilizados e citados pelos entrevistados foram a bota e o boné assim como observado por Castro et al. (2011).

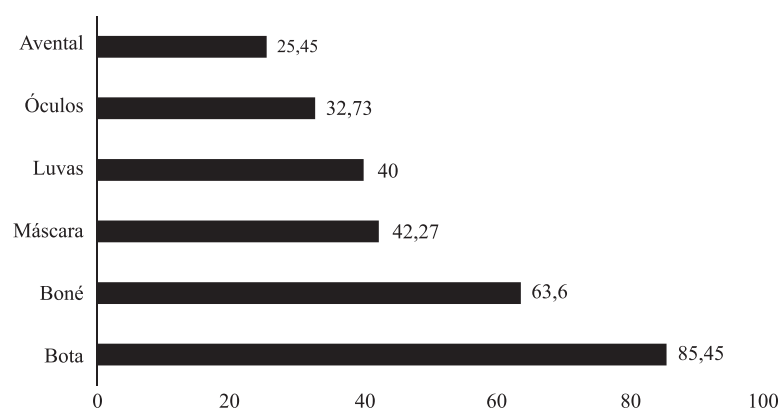

Figura 1 - Equipamentos de proteção individual utilizados pelos agricultores.
Não existe uma conscientização da importância da utilização do EPI completo por parte dos produtores, os quais afirmam não utilizarem devido ao desconforto e calor. Essa justificativa foi observada também por Zorzetti et al. (2014) na mesorregião do Norte Central do Paraná. Bedor et al. (2009) ressalta ainda que a falta de fiscalização no acompanhamento técnico e no controle de agrotóxicos faz com que a tomada de decisão do agricultor não leve em conta fatores relativos à saúde e ao meio ambiente, mas somente seja baseada na produtividade.

Essa crítica sobre o incômodo em trabalhar com o EPI também foi relatada em diversas entrevistas com agricultores que realizam a aplicação de produtos químicos, sendo um problema tecnológico conhecido, sendo válido ressaltar a atenção demandada para os fornecedores investirem até mesmo um modelo mais confortável que permita o uso completo desse equipamento (De Lima et al., 2009; Monqueiro et al., 2009; Marques et al., 2010).

Os locais de maior aquisição de agrotóxicos citados foram as lojas agropecuárias, onde aproximadamente $85 \%$ dos produtores consultaram um engenheiro agrônomo da loja na hora da compra, e os outros $15 \%$ fazem uso por conta própria. Dados semelhantes foram observados por Fernandes et al. (2012) na cidade de Ibirité/MG, onde 53,8\% (14) dos entrevistados afirmaram recorrer a engenheiros agrônomos ou técnicos e 46,2\% (12) afirmaram recorrer a uma casa agropecuária.

Essa assistência técnica no momento da compra de agrotóxicos é a única recebida pelos agricultores durante o ciclo da cultura, mostrando uma carência de orientação técnica por parte dos órgãos públicos e condicionando uma dependência dos produtores a agrônomos ligados aos próprios estabelecimentos de vendas desses produtos.

É essencial o conhecimento referente à classificação dos agrotóxicos quanto à sua ação e ao grupo químico para facilitar o diagnóstico em caso de intoxicações e também para fazer a rotação de diferentes grupos a fim de evitar o desenvolvimento de resistência a esses produtos. A Anvisa classifica produtos agroquímicos em quatro classes de risco à saúde. Cada classe é representada por uma cor diferente da etiqueta e pelo manual de instruções. A portaria IBAMA 84, de 15 de outubro de 1996, define quatro classes de risco ambiental para os produtos agroquímicos: Classe I: altamente perigoso; Classe II: muito perigoso; Classe III: perigoso e Classe IV: pouco perigoso.

A classificação toxicológica, o grupo químico do agrtóxico usado, o período de carência, assim como outras informações não eram de conhecimento da maioria 
dos entrevistados, pois, dentre eles, apenas 30\% afirmaram ler a bula ou o rótulo. Dados semelhantes foram relatados por Zorzetti et al. (2014) na mesorregião do Norte Central Paranaense.

Esse fato pode ser explicado pelo grau de escolaridade dos produtores de São José de Ubá, observouse que $71 \%$ possuíam o ensino fundamental incompleto, $18 \%$ o fundamental completo, $4 \%$ o ensino médio e $7 \%$ analfabetos. Segundo Oliveira-Silva et al., (2000), a baixa escolaridade pode afetar a leitura e entendimento sob as bulas dos agrotóxicos, e ainda impedir que adotem novas tecnologias de manejo, e possam realizar análises dos custos e viabilidade econômica da produção, e os fatores socioeconômicos, como o nível educacional, a habilidade de leitura e escrita e a renda familiar também podem estar relacionados ao impacto da contaminação por agrotóxicos.

De acordo com Vinha et al. (2011), alguns dos fatores associados às intoxicações por agrotóxicos em trabalhadores rurais são o uso indiscriminado, a propaganda massiva, o medo de perdada produção da safra, a cultura do "produto bonito",ou seja, aquele que o consumidor gosta de comprar,a não utilização dos equipamentos de proteção e opouco conhecimento dos riscos.

Entre os inseticidas, os produtos mais utilizados foram: $\operatorname{Actara}^{\circledR}(93 \%)$, Vertimec ${ }^{\circledR}(92 \%)$ e Evidence ${ }^{\circledR}(80 \%)$ (Tabela 1). Segundo a classificação toxicológica, 25\% dos utilizados são classificados como extremamente tóxico, $18,75 \%$ são altamente tóxico, $37,5 \%$ são mediamente tóxico e $18,75 \%$ pouco tóxicos.

Tabela 1 - Relação de inseticidas verificados na cultura do tomate no município de São José de Ubá, 2015

\begin{tabular}{|c|c|c|c|c|}
\hline Nome comercial & Ingrediente ativo & $\mathrm{TH}$ & TA & FR \\
\hline Actara $250 \mathrm{WG}$ & Neonicotinóide & III & III & $93 \%$ \\
\hline Ampligo & Piretróide e Antranilamida & II & I & $40 \%$ \\
\hline Arrivo $200 \mathrm{EC}$ & Piretroide & III & III & $64 \%$ \\
\hline Decis $25 \mathrm{EC}$ & Piretróide & III & I & $65 \%$ \\
\hline Engeo Pleno & Neonicotinóide e Piretróide & III & I & $32 \%$ \\
\hline Evidence & Neonicotinóide & IV & III & $80 \%$ \\
\hline Fastac 100 & Piretróide & II & I & $22 \%$ \\
\hline Karate 50 EC & Piretróide & II & I & $74 \%$ \\
\hline Kraft 36 EC & Avermectina & I & II & $45 \%$ \\
\hline Lannate BR & Metilcarbamato de oxima & I & II & $18 \%$ \\
\hline Orthene $750 \mathrm{BR}$ & Organofosforado & IV & III & $84 \%$ \\
\hline Potenza Sinon & Vermectinas & I & III & $26 \%$ \\
\hline Premio & Antranilamida -Diamida Antranílica & III & II & $65 \%$ \\
\hline Tiger $100 \mathrm{EC}$ & Éterpiridiloxipropílico & I & II & $74 \%$ \\
\hline Trigard $750 \mathrm{WP}$ & Triazinamina & IV & III & $61 \%$ \\
\hline Vertimec $18 \mathrm{EC}$ & Avermectina & III & II & $92 \%$ \\
\hline
\end{tabular}

TH - Toxidade humana; TA - Toxidade ambiental; FR - Frequência relativa.

Todos os agricultores usam Actara $^{\circledR}$ durante a produção de mudas para controlar Myzus persicae, Bemisia argentifolii e Thrips palmi. O inseticida Evidence ${ }^{\mathbb{R}}$ também é amplamente utilizado contra a mosca branca (Bemisia tabaci), mesmo que seu uso seja recomendado apenas para o cultivo de tabaco e cana-de-açúcar. O uso desses agrotóxicos é um dos recursos mais utilizados pelos produtores rurais para reduzir a incidência de pragas e doenças. Em contrapartida, há outras possíveis práticas adotadas, como o emprego da nutrição mineral adequada, com o uso de silício, com potencial para diminuir o uso de agroquímicos e aumentar a produtividade, conforme apontado por Santos et al. (2013) e Silva et al. (2010). 
Os fungicidas mais frequentes são Manzate (90\%) e Ridomil Gold Mz (85\%), em grande parte são utilizados para o controle de Phytophthora infestans. Os mesmos produtos são amplamente utilizados no município de Cambuci, noroeste do Rio de Janeiro, conforme afirmam Carvalho et al. (2016). A respeito da classificação toxicológica, constatou-se que $37,5 \%$ dos produtos utilizados são classificados como extremamente tóxico, $25 \%$ são altamente tóxico, e $37,5 \%$ são mediamente tóxico (Tabela 2).

Tabela 2 - Relação de fungicidas verificados na cultura do tomate no município de São José de Ubá, 2015

\begin{tabular}{|c|c|c|c|c|}
\hline Nome comercial & Ingrediente ativo & $\mathrm{TH}$ & $\mathrm{TA}$ & FR \\
\hline Bravonil Ultrex & Clorotalonil & I & II & $45 \%$ \\
\hline Cercobin $700 \mathrm{WP}$ & Tiofanato- metilico & II & III & $40 \%$ \\
\hline Cuzarte BR & Mancozebe & III & III & $67 \%$ \\
\hline Ridomil Gold Mz & Mancozebe & III & II & $85 \%$ \\
\hline Revus & Mandipropamida & II & IV & $70 \%$ \\
\hline Dithane NT & Mancozebe & I & II & $58 \%$ \\
\hline Manzate 800 & Mancozebe & I & II & $90 \%$ \\
\hline Kasumim & Casugamicina & III & III & $35 \%$ \\
\hline
\end{tabular}

TH - Toxidade humana; TA - Toxidade ambiental; FR - Frequência relativa.

O uso de agrotóxicos sem orientação adequada foi abordado por Fonseca et al. (2007) onde bulas, rótulos, televisão e rádio são apontados como fonte de informação pelos agricultores no que diz respeito a escolha de qual agrotóxico utilizar, bem como no que diz respeito a como proceder no manejo do agrotóxico. A literatura aponta que programas de capacitação do trabalho rural exercem grande impacto nos índices de morbi-mortalidade das intoxicações por agrotóxicos.

Apesar desse expressivo gasto com agrotóxicos, a maioria dos entrevistados acreditam que a produção orgânica seria uma cultivo sem a utilização de qualquer defensivo agrícola. Mais da metade dos produtores (56\%) afirmaram já terem ouvido falar da produção orgânica, mas desconhecem os produtos alternativos para controle e prevenção de pragas e doenças, isso inclui os $11 \%$ que já participaram de cursos sobre produção ecologicamente sustentável.

\section{CONCLUSÕES}

O uso de agrotóxicos nas lavouras de tomate é o manejo fitossanitário mais utilizado, apontando a carência de difusão de outras práticas de controle de pragas e doenças, como manejo nutricional, manejo integrado de pragas e avaliação de dano econômico para o uso mais racional de agrotóxicos.
Falta maior conscientização para um manuseio seguro e instruções sobre os perigos que os agrotóxicos oferecem, e a maneira correta de utilizá-los a fim de evitar acidentes e danos ao meio ambiente.

Esse trabalho indica a necessidade de ações conjuntas entre a Secretaria de Agricultura, Emater, lojas agropecuárias e os tomaticultores, através de dia de campo, palestras de gerenciamento financeiro dos agrotóxicos e educação ambiental.

\section{LITERATURA CITADA}

ALMEIDA, V.E.S.; CARNEIRO, F.F.; VILELA, N.J. Agrotóxicos em hortaliças: segurança alimentar, riscos socioambientais e políticas públicas para promoção da saúde. Tempus Actas de Saúde Coletiva, v.4, n.4, p.84-99, 2009.

BEDOR, C.N.G.; RAMOS, L.O.; PEREIRA, P.J.; RÊGO, M.A.V.; PAVÃO, A.C.; AUGUSTO, L.G.D.S. Vulnerabilidades e situações de riscos relacionados ao uso de agrotóxicos na fruticultura irrigada. São Paulo. Revista Brasileira de Epidemiologia, v.12, n.1, p.39-49, 2009.

CAMARGO, F.P. de; FILHO, W.P.C. Produção de tomate de mesano Brasil, 1990-2006: contribuição da área e da produtividade. Horticultura Brasileira, v.26, n.2, p.S1018-S1021, 2008. 
CARVALHO, C.R.F.; PONCIANO, N.J; SOUZA, C.L.M de. Levantamento dos agrotóxicos e manejo na cultura do tomateiro no município de Cambuci - RJ. Ciência Agrícola, v.14, n.1, p.15-28, 2016.

CASTRO, M.G.G.M.; FERREIRA, A.P.; MATTOS, I.E. Uso de agrotóxicos em assentamentos de reforma agrária no Município de Russas (Ceará, Brasil): um estudo de caso. Epidemiologia e Serviços de Saúde, v.20, n.2, p.245-254. 2011.

DE LIMA, C.A.B.; GRÜTZMACHER, D.D.; KRÜGER, L.R.; GRÜTZMACHER, A.D. Diagnóstico da exposição ocupacional a agrotóxicos na principal região produtora de pêssego para indústria do Brasil. Ciência Rural, Santa Maria, v.39, n.3, p.900-903, 2009.

DURIGUETTO, P.S.; RAMOS; F.O.; DE OLIVEIRA MARQUES, E.; ARRUDA, V. M. Diagnóstico do desenvolvimento da agricultura alternativa no município de Ubá_MG. Revista Brasileira de Agropecuária Sustentável, v.3, n.1, p.1-10, Julho, 2013.

FERNANDES, A.A.; MARTINEZ, H.E.P.; DA SILVA, D.J.H.; BARBOSA, J.G.; PEDROSA, A.W. Cultivo sucessivo de plantas de tomate oriundas de sementes e propagação vegetativa em sistema hidropônico. Pesquisa Agropecuária Brasileira, v.42, n.7, p.1013-1019, 2007.

FERNANDES, V.A.; SILVA, L.F.; MESQUITA, T.R.R.; CAPETTINI, L.S.A.; RODRIGUES, A.L.P.; SANTOS, S.L. Uso de pesticidas na agricultura - Análise da prática na cidade de Ibirité/MG. Scientia Plena, v.8, n.3, p.1-6, 2012.

FONSECA, M.G.U.; PERES, F.; FIRMO, J.O.A.; UCHOA, E. Percepção de risco: maneiras de pensar e agir no manejo de agrotóxicos. Ciência e Saúde Coletiva, v.12, n.1, p.39$50,2007$.

IBGE - INSTITUTO BRASILEIRO DE GEOGRAFICA E ESTATÍSTICA. Relatório da produção de lavouras temporárias dos anos 2014 a 2018. Disponível em: $<\quad$ http://seriesestatisticas.ibge.gov.br/lista tema. aspx?op=0\&no=1> Acessado em: 05/01/2019.

LIMA, K.K.P.S.; CAMPOS, K.C. Viabilidade financeira do tomate convencional e orgânico no estado do Ceará. Revista Sociais e Humanas, v.27, n.2, p.26-39, 2014.

MARQUES, C.R.G.; NEVES, P.M.O.J.; VENTURA, M.U. Diagnóstico do conhecimento de informações básicas para o uso de agrotóxicos por produtores de hortaliças da Região de Londrina. Semina: Ciências Agrárias, v.31, n.3, p.547-556, 2010.

MONQUEIRO, P.A.; INÁCIO, E.M.; SILVA, A.C. Levantamento de agrotóxicos e utilização de equipamento de proteção individual entre os agricultores da região de Araras. Arquivos do Instituto Biológico, v.76, n.1, p.135139, 2009.

MONTORO, A.E.P.; BRANCO JUNIOR, A.C. Perfil de Produtores rurais quanto ao uso de defensivos agrícolas no interior do Estado de São Paulo. In: Congresso Brasileiro de Gestão Ambiental. 2013. Anais. Salvador/BA: Ibeas, p.1-5, 2009.

OLIVEIRA-SILVA, J.J.; ALVES, S.R.; MEYER, A., PEREZ, F.; SARCINELLI, P.D.N.; DA MATTOS, R.D.C.O.; MOREIRA, J.C. Influência de fatores socioeconômicos na contaminação por agrotóxicos, Brasil. Revista de Saúde Pública, v.35, n.2, p.130-135, 2001.

PEREIRA, R.B.; PINHEIRO, J.B.; DE CARVALHO, A.D.F. Diagnose e controle alternativo de doenças em tomate, pimentão, curcurbitáceas e cenoura. Brasília, DF: EMBRAPA HORTALIÇAS, 2013. 16p. (Circular Técnica, 121)

REIS FILHO, J.D.S.; MARIN, J.O.B.; FERNANDES, P.M. Os agrotóxicos na produção de tomate de mesa na região de Goianápolis, Goiás. Pesquisa Agropecuária Tropical, v.39, n.4, p.307-316, 2009.

SANTOS, M.C., DE FREITAS, L.M. Efeito do silício, nitrogênio e potássio na incidência da traça-do-tomateiro em plantas para processamento industrial. Revista Brasileira de Agropecuária Sustentável, v.3, n.2, p.19-24, 2013.

SILVA, V.F.; MORAES, J.C.; MELO, B.A. Influenceof silicon on the development, productivity andinfestation by insect pests in potato crops. Ciência e Agrotecnologia, v.34, p.1465-1469, 2010.

STEVENSON, W.J. Estatística Aplicada à Administração. 1.ed. São Paulo: Habra. 2001.

VINHA, M.B.; DE OLIVEIRA PINTO, C.L.; PINTO, C.M.F.; DE SOUZA, C.F.; DE MIRANDA SOUZA, M.R.; DE OLIVEIRA, L.L. Impactos do uso indiscriminado de agrotóxicos em frutas e hortaliças. Revista Brasileira de Agropecuária Sustentável, v.1, n.1, p.102-107, Julho, 2011.

ZORZETTI, J.; OLIVEIRA, M.J.N.; SANTORO, P.H.; CONSTANSKI, K.C. Conhecimento sobre a utilização segura de agrotóxicos por agricultores da mesorregião do Norte Central do Paraná. Semina: Ciências Agrárias, v.35, n. $4,2014$.

Recebido para publicação em 26/06/2019, aprovado em 03/04/2020 e publicado em 30/04/2020. 